\title{
Superimposed Infectious Colitis in Crohn's Disease
}

\author{
Matthew A. Crain ${ }^{a} \quad$ Justin T. Kupec ${ }^{b}$ \\ aWest Virginia University School of Medicine, Morgantown, WV, USA; 'bection of \\ Gastroenterology and Hepatology, West Virginia University Medicine, Morgantown, WV, \\ USA
}

\author{
Keywords \\ Crohn's disease - Plesiomonas shigelloides - Enteric infection · Microbial stool testing · \\ Differential diagnosis
}

\begin{abstract}
The differential diagnosis for an acute Crohn's flare should include enteric infection, a challenging yet critical distinction to make when determining appropriate therapy. Since both present similarly, identification of an enteric infection should be performed with comprehensive stool microbial testing. In the setting of moderate-to-severe disease, patients on biologic therapy may be more prone to infectious complications. We present a patient with chronic Crohn's disease with an unusual, previously undetected enteric infection due to Plesiomonas shigelloides. Once identified, appropriate antibiotic treatment led to resolution of the patient's acute symptomatology. This is the first reported case of $P$. shigelloides infection in Crohn's disease.
\end{abstract}

\section{Introduction}

Crohn's disease is a relapsing inflammatory bowel disease characterized by abdominal pain, fever, bowel obstruction, and diarrhea. Patients with Crohn's disease often present with repeated acute flares, requiring urgent care and, at times, hospitalizations. The development of biologic therapy has dramatically improved clinical outcomes of patients with moderate-to-severe Crohn's disease but has had unintended immunological consequences. In particular, biologics can lead to increased susceptibility to infections, which can become serious in an immunocompromised patient with Crohn's disease. Unfortunately, it can be difficult to distinguish between a relapse of Crohn's disease and superimposed infectious colitis based upon presentation of symptoms. We describe a patient in which an unusual underlying enteric infection went undetected for months 
despite frequent outpatient, emergency, and hospital care, leading to an exacerbation and prolongation of serious gastrointestinal symptoms. The case illustrates the importance of promptly identifying the existence of enteric infections that may present with symptoms that mimic Crohn's disease relapse in order to treat with appropriate therapies.

\section{Case Presentation}

A 24-year-old male with 6 years of moderate-to-severe fistulizing Crohn's disease presented as an outpatient with 6 months of bloody diarrhea, left lower quadrant abdominal pain, 15-pound weight loss (from 141 to 126 pounds), and fever (to $103^{\circ} \mathrm{F}$ ). Currently on vedolizumab, the patient had previously been treated unsuccessfully with adalimumab and infliximab. He had been on metronidazole for perianal abscesses. Recent colonoscopy demonstrated persistent ulceration in the rectosigmoid colon which was confirmed with CT. Despite aggressive outpatient management with the addition of prednisone to vedolizumab and metronidazole, his symptoms persisted. Upon admission for failed outpatient management, the patient received IV corticosteroids, hydration, and pain control in addition to evaluation for infectious colitis. Clostridioides difficile testing was negative, but the BioFire FilmArray gastrointestinal (GI) panel was positive for Plesiomonas shigelloides.

Ciprofloxacin was initiated for suspected superimposed infectious colitis from $P$. shigelloides (likely obtained from fresh water exposure due to swimming in a local river), and he was instructed to taper off prednisone. Within a few days, the patient's symptoms reduced significantly, and at 2 weeks, he indicated that his abdominal pain and diarrhea were significantly improved. Due to ongoing Crohn's, his treatment plan will be altered in upcoming clinic visits.

\section{Discussion and Conclusion}

Biologic agents such as adalimumab and vedolizumab have dramatically improved the treatment of Crohn's disease, leading to remission or at least reduction of symptoms in most patients [1]. Unfortunately, biologic therapy has also produced unintended immunological consequences, potentially leading to vulnerability to serious opportunistic infections as well as other adverse effects [2].

While early reports suggested enteric infection is rare in IBD, and routine microbiological stool testing not necessary, newer data shed more light on the role of infection complicating the care of patients with IBD [3]. A 5-year retrospective study of stool samples in patients with undifferentiated IBD taken during a relapse determined that $>10 \%$ of 213 patients had enteric infections. More recently, the literature supports the expanded use of BioFire FilmArray GI panel [4] for stool microbial PCR testing [5, 6]. In a review of records of 214 IBD patients experiencing an exacerbation of symptoms, including 103 patients with Crohn's disease, nearly 27\% were positive for pathogens [5]. Only 1 patient tested positive for $P$. shigelloides (there was no differentiation between IBD types). Another study with a larger sample size confirmed a higher prevalence of norovirus and Campylobacter relative to patients without IBD, which could not be differentiated by endoscopic and histological findings and may play an important role in flares [6]. P. shigelloides, present in several ulcerative colitis patients, was not seen in a single Crohn's patient. Finally, a recent systematic review revealed that certain enteric pathogens may be associated with an increased risk of developing IBD including Crohn's disease [7].

For patients with IBD, it is often difficult to distinguish between an enteric infection and symptoms of a flare $[8,9]$. When a pathogen is identified, targeted therapy should be administered. Focusing only on treating, the underlying IBD may lead to prolonged exacerbation of symptoms.

\section{Karger'}


P. shigelloides is a pathogen found in freshwater ecosystems. While not likely to trigger a serious infection under normal circumstances, immunosuppression (e.g., biologic therapy to treat IBD) may result in a prolonged course. P. shigelloides is often overlooked in stool samples due to its smaller colony size and relatively low prevalence in gastrointestinal samples [10]. The BioFire FilmArray GI panel is the first FDA-approved PCR-based culture-independent diagnostic test system that includes $P$. shigelloides in its panel [4].

In conclusion, we present to you the first reported case of $P$. shigelloides infection in Crohn's disease. This case highlights both the importance of ruling out and treating infectious colitis as a cause of worsened symptoms in patients with IBD as well as understanding the unique role of immunosuppression in this patient population.

\section{Statement of Ethics}

Written informed consent was provided by the patient to publish his case. The authors verify that this is their own, previously unpublished, original work.

\section{Conflict of Interest Statement}

The authors have no conflicts of interest or financial disclosures to declare.

\section{Funding Sources}

There were no funding sources for the preparation of this manuscript.

\section{Author Contributions}

Both authors were equally responsible for this manuscript with M.C. responsible for concept, data collection, manuscript generation, and submission and J.K. responsible for editing and manuscript generation.

\section{References}

1 Baumgart DC, Sandborn WJ. Crohn's disease. Lancet. 2012;380(9853):1590-605.

2 Henrickson SE, Ruffner MA, Kwan M. Unintended immunological consequences of biologic therapy. Curr Allergy Asthma Rep. 2016;16(6):46-7.

3 Mylonaki M, Langmead L, Pantes A, Johnson F, Rampton DS. Enteric infection in relapse of inflammatory bowel disease: importance of microbiological examination of stool. Eur J Gastroenterol Hepatol. 2004;16(8):775-8.

4 Machiels JD, Cremers AJH, van Bergen-Verkuyten MCGT, Paardekoper-Strijbosch SJM, Frijns KCJ, Wertheim $\mathrm{HF}$, et al. Impact of the BioFire FilmArray gastrointestinal panel on patient care and infection control. PLoS One. 2020;15(2):e0228596.

5 Axelrad JE, Joelson A, Nobel YR, Lawlor G, Green PHR, Lichtiger S, et al. Enteric infection in relapse of inflammatory bowel disease: the utility of stool microbial PCR testing. Inflamm Bowel Dis. 2017;23(6):1034-9.

6 Axelrad JE, Joelson A, Green PHR, Lawlor G, Lichtiger S, Cadwell K, et al. Enteric infections are common in patients with flares of inflammatory bowel disease. Am J Gastroenterol. 2018;113:1530-9.

7 Axelrad JE, Cadwell KH, Colombel J-F, Shah SC. Systematic review: gastrointestinal infection and incident inflammatory bowel disease. Aliment Pharmacol Ther. 2020;51:1222-32.

8 Kucharzik T, Maaser C. Infections and chronic inflammatory bowel disease. Viszeralmedizin. 2014;30:326-32.

9 De Hertogh G, Geboes K. Crohn's disease and infections: a complex relationship. Med Gen Med. 2004;6(3):1-13.

10 Janda JM, Abbott SL, McIver CJ. Plesiomonas shigelloides revisited. Clin Microbiol Rev. 2016;29:349-74.

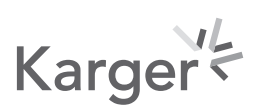

